\title{
Simulation of collagen solution flow in rectangular capillary
}

\author{
Bohus Kysela ${ }^{1}$, Jan Skocilas², Rudolf Zitny ${ }^{2}$, Jaromir Stancl $^{2}$, Milan Houska $^{3}$, and Ales Landfeld ${ }^{3}$ \\ 1 Institute of Hydrodynamics, AS CR, v.v.i., Pod Patankou 30/5, 16612 Prague 6, Czech Republic \\ 2 Department of Process Engineering, Faculty of Mechanical Enginnering, Czech Technical University in Prague, \\ Technicka 4, 16607 Prague 6, Czech Republic \\ 3 Food Research Institute Prague, Radilova 7, 10200 Prague 10, Czech Republic
}

\begin{abstract}
The viscoelastic properties of foods and polymers can be evaluated from flow of the material in capillary with specified dimension and shape. The extrusion rheometer equipped by capillary with rectangular cross-section was used for determination of the rheological behaviour of water collagen solution. The measurements of the axial profiles in longitudinal direction of the total stresses at capillary wall were performed for various shear rates. The linear viscoelastic model of Oldroyd B type: White-Metzner model was used for simulation of fluid flow in OpenFOAM software package. The simulations describe the effect of relaxation time on wall total stress in convergent-divergent capillary.
\end{abstract}

\section{Introduction}

The aim of this study is to solve the flow of viscoelastic fluid (water collagen solution) using the OpenFOAM - extended with application of "viscoelasticFoam" solver and its modifications. This solver was developed and implemented by Favero at al. [1]. The main goal is to obtain dependency of total normal stress $\sigma$ on bottom wall of the rectangular capillary:

$$
\sigma=\tau_{y y}-p
$$

where $\tau_{y y}$ is normal extrastress component and $p$ is pressure inducted by the flow of viscoelastic fluid. Rheological properties are modelled by the constitutive model WhiteMetzner [2] combined with power law fluid model.

In this study the capillary with convergent-divergent part was chosen for investigation of relaxation time effect on wall total stress. This simple geometry consist of stabilizing part $200 \mathrm{~mm}$, convergent part $200 \mathrm{~mm}$, and divergent part $200 \mathrm{~mm}$ (see fig. 1). On base of the previous exper-

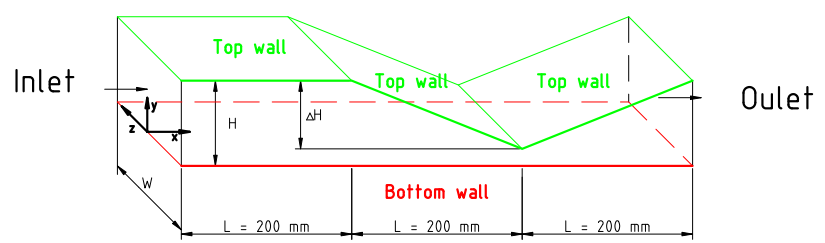

Figure 1. Sketch of geometry which was used for creation of computational domain. $(H=5 \mathrm{~mm}, \Delta H=4 \mathrm{~mm}, L=$ $200 \mathrm{~mm}, W=8 \mathrm{~mm}$ ).

imental investigations $[3,4]$ material constants were proposed for used water collagen solution, that are necessary as input to computational model. The proposed material properties for that case were: density $\rho=1000 \mathrm{~kg} \cdot \mathrm{m}^{-3}$;
Newtonian solvent viscosity $\eta_{s}=7 \mathrm{~Pa} \cdot s$; coefficient of consistency $k=1353 \mathrm{~Pa} \cdot \mathrm{s}^{n}$; flow index $n=0.25$; the relaxation time varied from $\lambda=0.003$ to $0.1 s$ (for water $\lambda \approx 10^{-12} \mathrm{~s}$ ).

\section{Theory of solution method}

The method was originally implemented on finite volume method based on solving following system of equations: Continuity equation:

$$
\nabla \cdot(U)=0
$$

Momentum equation:

$$
\frac{\partial(\rho U)}{\partial t}+\nabla \cdot(\rho U U)=-\nabla p+\nabla \cdot \tau_{s}+\nabla \cdot \tau_{p},
$$

where $\tau_{s}$ is shear tensor acting from Newtonian solution and $\tau_{p}$ is extra elastic shear tensor.

$$
\tau_{s}=2 \eta_{s} D
$$

where $\eta_{s}$ is viscosity of Newtonian solution and $D$ is strain rate tensor:

$$
D=\frac{1}{2}\left(\nabla U+[\nabla U]^{T}\right)
$$

Constitutive model White-Metzner:

$$
\tau_{p_{K}}+\lambda_{K}\left(I I_{D}\right) \stackrel{\nabla}{\tau_{p_{K}}}=2 \eta_{p_{K}}\left(I I_{D}\right) D
$$

where $\stackrel{\nabla}{\tau}_{p_{K}}$ represents upper convected derivatives and second invariant of strain rate tensor is

$$
\left(I I_{D}\right)=\dot{\gamma}=\sqrt{2 D: D} .
$$

For power law model it was defined:

$$
\eta_{p_{K}}\left(I I_{D}\right)=k\left(I I_{D}\right)^{n-1}
$$

and relaxation time was defined as constant value:

$$
\lambda_{K}\left(I I_{D}\right)=\lambda=\text { const } .
$$


Table 1. Boundary conditions.

\begin{tabular}{cccc}
\hline Boundary & Velocity & Pressure & Tau \\
\hline Inlet & $\left(\begin{array}{lll}0.1 & 0 & 0\end{array}\right)$ & zeroGradient & $\left(\begin{array}{lll}0 & 0 & 0\end{array}\right)$ \\
Outlet & zeroGradient & 0.0 & zeroGradient \\
Walls & $\left(\begin{array}{llll}0 & 0 & 0\end{array}\right)$ & zeroGradient & zeroGradient
\end{tabular}

Table 2. Material model constants of water.

\begin{tabular}{ccc}
\hline Name & Variable & Value \\
\hline Density & $\rho\left[\mathrm{kg} \cdot \mathrm{m}^{-3}\right]$ & 1000 \\
Newtonian solvent viscosity & $\eta_{s}[\mathrm{~Pa} \cdot \mathrm{s}]$ & 0.001 \\
Coefficient of consistency & $k\left[\mathrm{~Pa} \cdot \mathrm{s}^{n}\right]$ & 0.001 \\
Flow index & $n[-]$ & 1.0 \\
Relaxation time & $\lambda[\mathrm{s}]$ & $\approx 10^{-12}$ \\
\hline
\end{tabular}

Table 3. Material model constants of water collagen solution [4].

\begin{tabular}{ccc}
\hline Name & Variable & Value \\
\hline Density & $\rho\left[\mathrm{kg} \cdot \mathrm{m}^{-3}\right]$ & 1000 \\
Newt. solv. viscosity & $\eta_{s}[\mathrm{~Pa} \cdot \mathrm{s}]$ & 7.0 \\
Coef. of consistency & $k\left[\mathrm{~Pa} \cdot \mathrm{s}^{n}\right]$ & 1353 \\
Flow index & $n[-]$ & 0.25 \\
Relaxation time & $\lambda[\mathrm{s}]$ & $10^{-12} ; 0.003 ; 0.01 ; 0.1$ \\
\hline
\end{tabular}

\section{Simulation set-up and results}

The OpenFOAM-extended package v3.2 was used. The mesh $1000 \times 30 \times 20$ was generated and optimized by blockMesh utility. The boundary conditions are given in table 1 . The inlet is defined by constant velocity $U_{x}=$ $0.1 \mathrm{~m} \cdot \mathrm{s}^{-1}$ (where $x$ coordinate corresponds with capillary axis, while $y$ coordinate corresponds with the capillary height) and with zero shear. The coordinate system origin $(x, y, z)=(0,0,0)$ is set in the middle of the channel width at the bottom wall at velocity inlet. The task was divided into two parts, where the first one was focused on the validation of the solver results, and the second one on the flow simulations of water collagen solution with variable $\lambda$ values from $10^{-12}$ to $0.1 s$ (see table 3 ). The validation was performed with material constants of water (see tab. 2).

\subsection{Flow simulation with water properties}

The initial calculations were performed for basic verification of proposed model. Newtonian fluid (water) was used as basic fluid, where the relaxation time $\lambda \approx 10^{-12} s$ (see tab. 2). The velocity profiles and distribution of total shear stress evaluated perpendicularly to the axis of rectangular capillary in distance $0.2 \mathrm{~m}$ of length (in the end of the linear section) and in distance $0.4 \mathrm{~m}$ of length (in contraction) are depicted in figs. 2 and 3. The velocity profile obtained in the developing section (the first linear section) is parabolic in shape and it is in agreement with presumptions as well as the evaluated total shear stress values are in agreement.

Then the aim was to obtain the distribution of the total normal stress $\sigma$ along the capillary (eq. 1). The distribution of pressure and shear stress component $\tau_{y y}$ acting on bottom wall (see fig. 1) is depicted in fig. 4. The behaviour of pressure distribution is also in agreement with presumptions, where the pressure linearly decreased with length
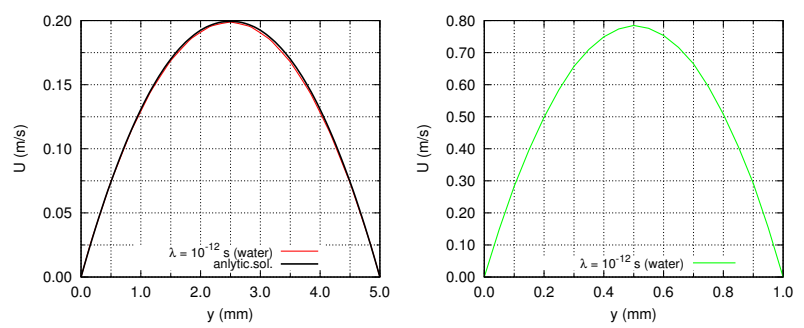

Figure 2. Velocity profiles in two distances $\mathrm{L}=0.2$ (left); 0.4 $\mathrm{m}$ (right) for water properties. The first profile is compared with analytical solution [5].
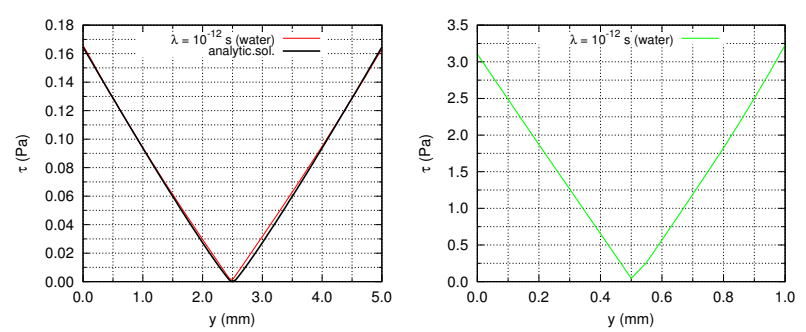

Figure 3. Profiles of shear stress for two distances $L=0.2$ (left); $0.4 \mathrm{~m}$ (right) for water properties. The first profile is compared with analytical solution [5].
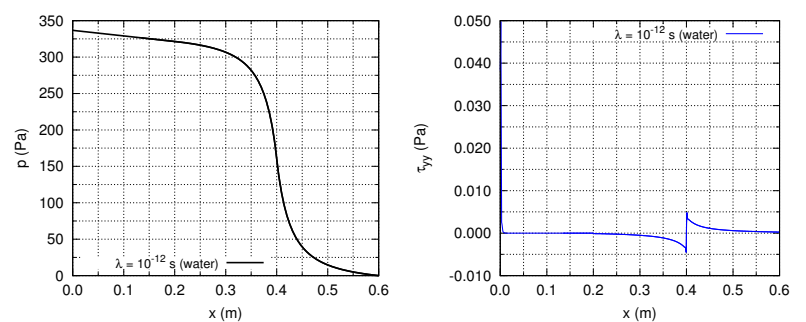

Figure 4. Evaluated pressure (left) and shear stress component $\tau_{y y}$ (right) acting on wall for "water" flow through capillary.

(in the first linear region). Then the convergent and divergent regions are represented by symmetrical pressure drop curves with inflection point in contraction point. Moreover, shear stress component $\tau_{y y}$ calculated from water properties seems to be negligible in comparison with pressure values.

\subsection{Results of collagen solution flow simulation}

The following calculations were performed with properties corresponding to experimental investigations [4]. The proposed parameters are given in tab. 3. The example of obtained distribution of pressure $p$ and shear stress component $\tau_{y y}$ acting on bottom wall (see fig. 1) is depicted in fig. 5 for relaxation time was $\lambda=0.003 s$. The velocity profiles and profiles of total normal stress for all simulated relaxation times in distance $0.2 \mathrm{~m}$ of length (in the end of the linear section) and in $0.4 \mathrm{~m}$ of length (in contraction) are depicted in figs. $6-7$ and 8-9.

The shapes of velocity profile in linear developing section are very similar and it seems that the relaxation time has no effect on its shape, while the profile in contraction shows the deformation. The evaluation of total shear stress indicates the increase of shear stress near walls with increasing relaxation time in both profiles. 

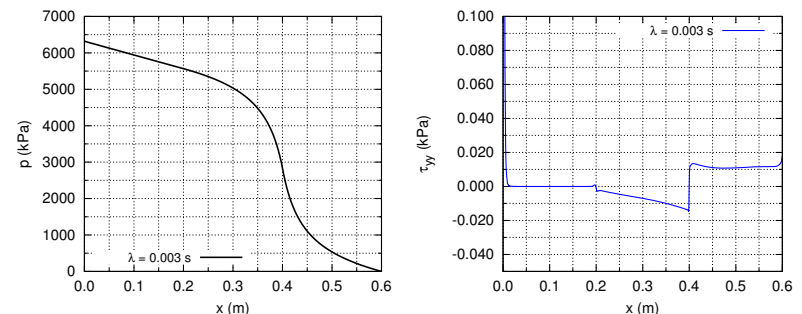

Figure 5. Evaluated pressure (left) and shear stress component $\tau_{y y}$ (right) on wall for flow of collagen solution through capillary: $\lambda=0.003 \mathrm{~s}$.

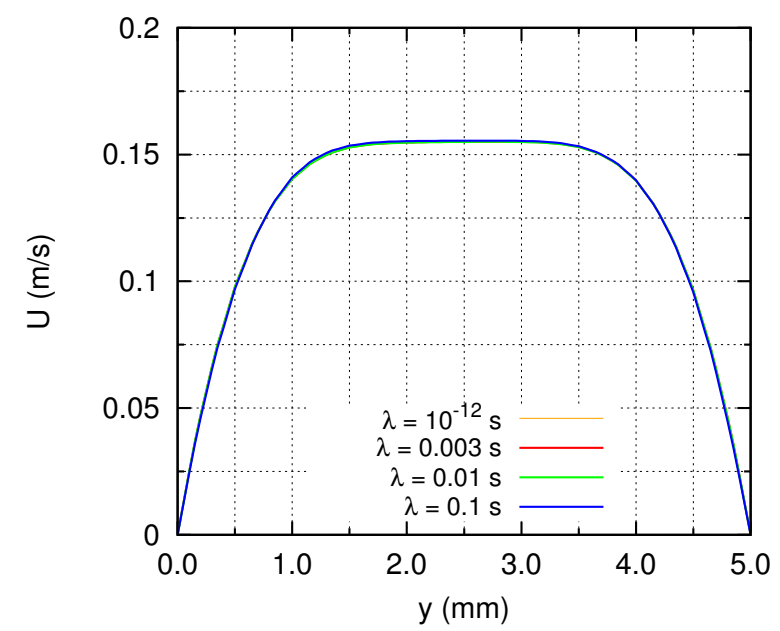

Figure 6. Velocity profile of collagen solution flow simulation $\left(\lambda=10^{-12} ; 0.003 ; 0.01 ; 0.1 s\right)$ in distance $\mathrm{L}=0.2 \mathrm{~m}$.

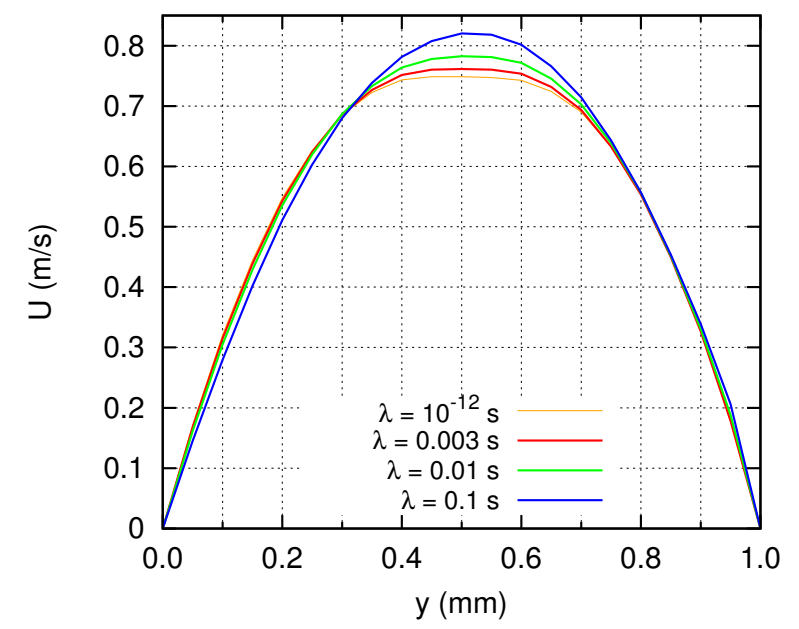

Figure 7. Velocity profile of collagen solution flow simulation $\left(\lambda=10^{-12} ; 0.003 ; 0.01 ; 0.1 s\right)$ in distance $\mathrm{L}=0.4 \mathrm{~m}$.

\subsection{Comparison of pressure and $\tau_{y y}$ dependencies acting on wall}

The distinct disproportion between values of pressure and shear stress component $\tau_{y y}$ was also found for collagen solution. This concludes that the shear stress component $\tau_{y y}$ is negligible in comparison with pressure values. The dependency on relaxation time is depicted in fig. 10 .

The shear stress $\sigma$ acting on capillary wall is proportional to the pressure. The distributions of pressure are not quite identical consequently the values were normalised by the

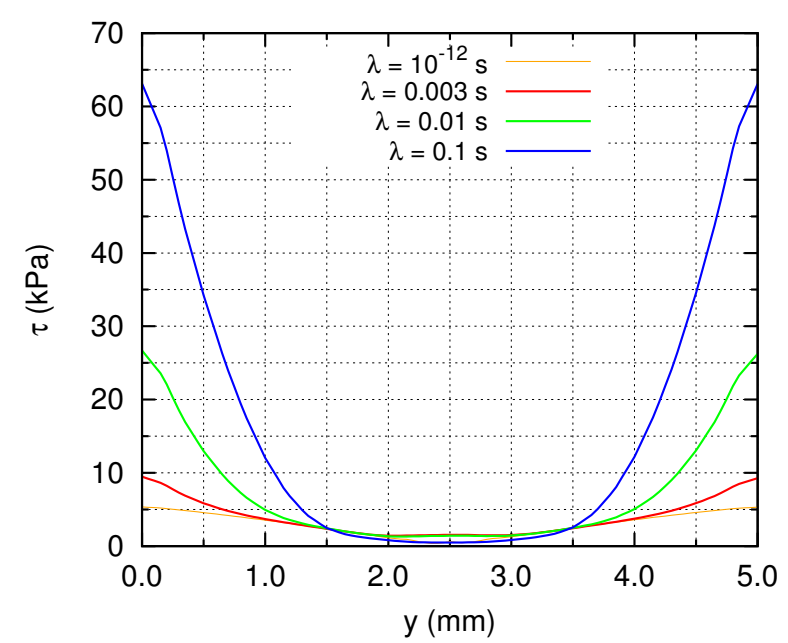

Figure 8. Profile of shear stress of collagen solution flow simulation $\left(\lambda=10^{-12} ; 0.003 ; 0.01 ; 0.1 s\right)$ in distance $\mathrm{L}=0.2 \mathrm{~m}$.

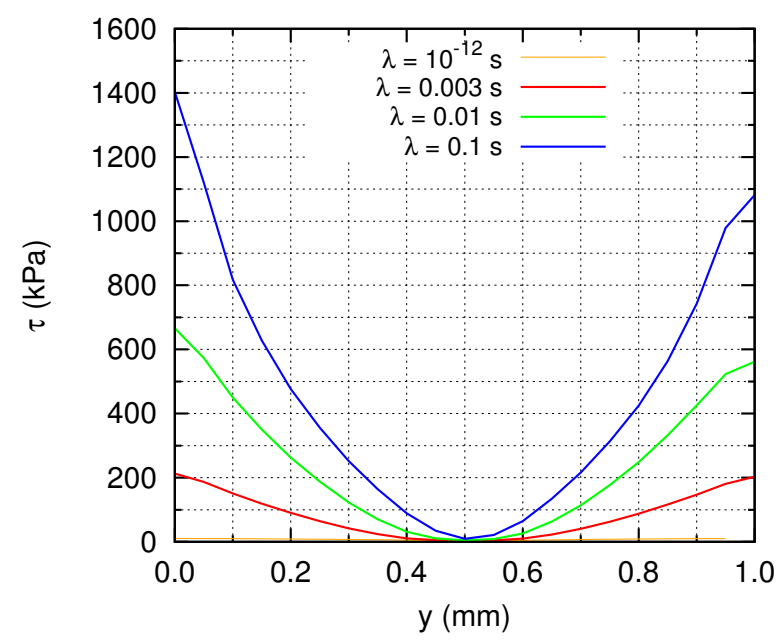

Figure 9. Profile of shear stress of collagen solution flow simulation $\left(\lambda=10^{-12} ; 0.003 ; 0.01 ; 0.1 s\right)$ in distance $\mathrm{L}=0.4 \mathrm{~m}$.

value $p_{0.2 m}$ where the developing linear section ends (see fig. 1). The normalized pressures acting on wall are shown in fig. 11. The discrepancy for water (the slope in the developing linear section) is given by parameters of fluid.

The effect of relaxation time and the deformation of the pressure curves in convergent and divergent sections is presented in fig. 12, where the detailed view onto contraction point $(\mathrm{L}=0.4 \mathrm{~m})$ shows the discrepancy and irregularity of pressure curves due to shift from point $p / p_{0.2 m}=0.5$ caused by the change of the relaxation time.

\section{Conclusions}

The proposed model of viscoelastic fluid was implemented and the calculation was validated for Newtonian fluid (data for water) in rectangular capillary. It could be assumed that the basic calculations of the flow are valid.

The obtained results indicate that the shear stress component $\tau_{y y}$ is negligible in comparison with pressure values for calculations of total stress $\sigma$ measured on wall of the capillary.

The effect of relaxation time is shown on comparison of 


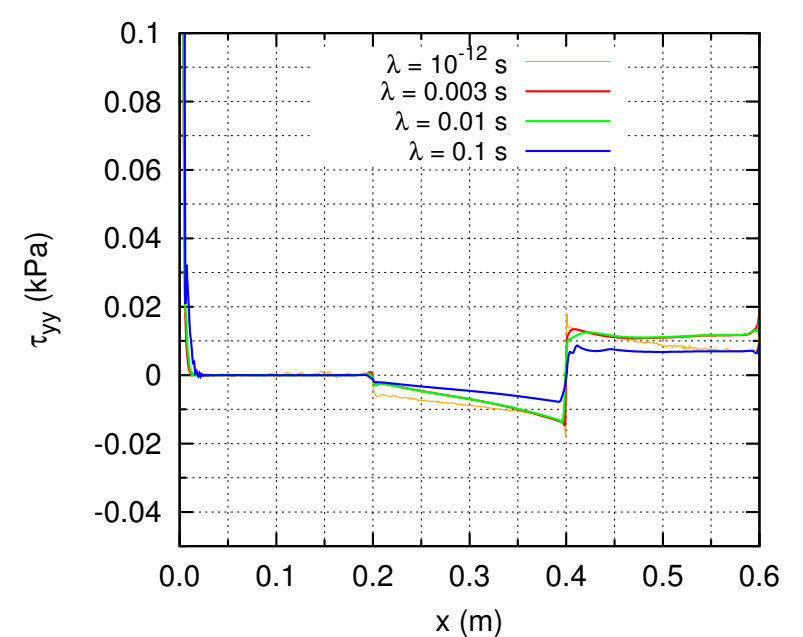

Figure 10. Shear stress component distributions $\tau_{y y}$ acting on wall $\left(\lambda=10^{-12} ; 0.003 ; 0.01 ; 0.1 s\right)$.

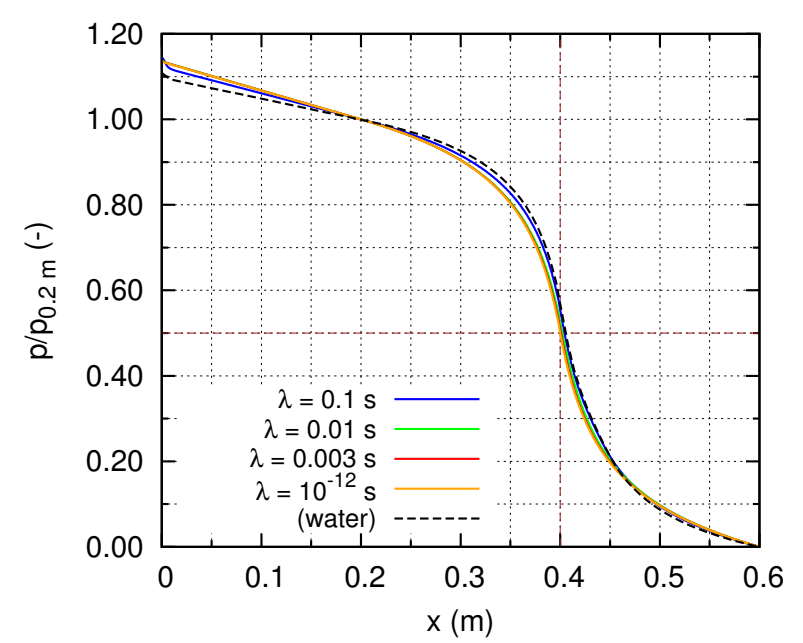

Figure 11. Comparison of the pressure distributions normalized by value $p_{0.2 m}$ i.e. pressure at the end of linear section where $\mathrm{L}=$ $0.2 \mathrm{~m}$ (see fig. 1).

the pressure dependency (fig. 11 and 12), where its shift in inflection point of pressure curve is proportional to the relaxation time. It indicates that the deformation of pressure curves and its irregularity for convergent and divergent channel section is caused by the relaxation time effect.

\section{Acknowledgments}

This research has been subsidized by the research project No. GA ČR 14-23482S and RVO: 67985874.

\section{References}

1. J. Favero, A. Secchi, N. Cardozo, H. Jasak, Journal of Non-Newtonian Fluid Mechanics 165, 1625 (2010)

2. J.L. White, A.B. Metzner, Journal of Applied Polymer Science 7, 1867 (1963)

3. R. Zitny, A. Landfeld, J. Skocilas, J. Stancl, V. Flegl, M. Houska, Acta Polytechnica 55, 352 (2015)

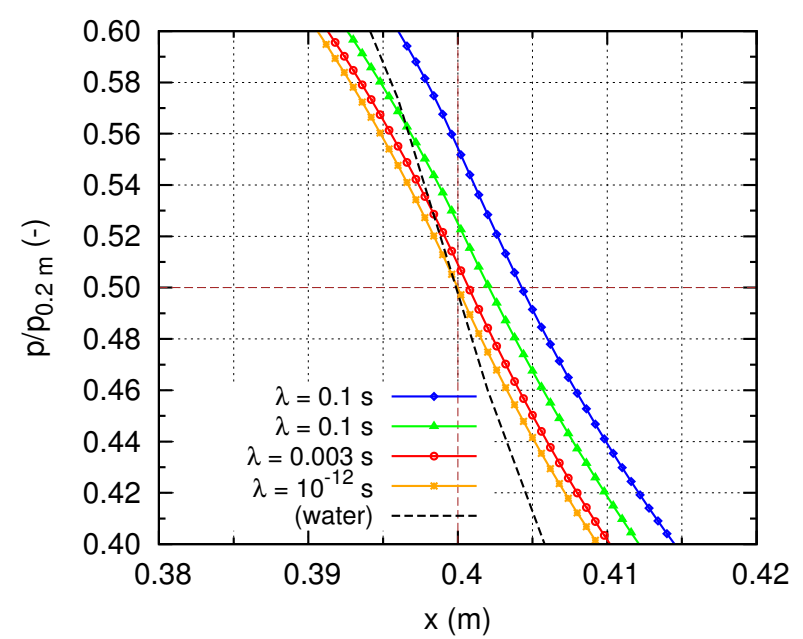

Figure 12. Comparison of the pressure distributions normalized by value $p_{0.2 m}$ i.e. pressure at the end of linear section where $\mathrm{L}=$ $0.2 \mathrm{~m}$ (see fig. 1). Detail in vicinity of inflexion point $(\mathrm{L}=0.4 \mathrm{~m}$; $\left.p / p_{0.2 m}=0.5\right)$.

4. J. Skočilas, R. Žitný, J. Štancl, M. Dostál, A. Landfeld, M. Houška, Journal of Texture Studies (2016), (in print)

5. R. Shah, A. London (Academic Press, 1978), ISBN 978-0-12-020051-1 